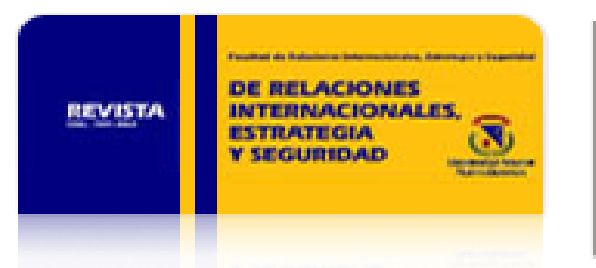

Revista de Relaciones Internacionales, Estrategia y Seguridad

ISSN: 1909-3063

cinuv.relinternal@unimilitar.edu.co

Universidad Militar Nueva Granada

Colombia

Delgado Barón, Mariana

RECONCEPTUALIZANDO LA SEGURIDAD: CAMBIO DE DILEMAS Y AMENAZAS

Revista de Relaciones Internacionales, Estrategia y Seguridad, vol. 3, núm. 2, julio-diciembre, 2008,

pp. 113-134

Universidad Militar Nueva Granada

Bogotá, Colombia

Disponible en: http://www.redalyc.org/articulo.oa?id=92712967006

- Cómo citar el artículo

- Número completo

- Más información del artículo

Página de la revista en redalyc.org

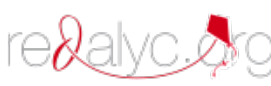

Sistema de Información Científica

Red de Revistas Científicas de América Latina, el Caribe, España y Portugal

Proyecto académico sin fines de lucro, desarrollado bajo la iniciativa de acceso abierto 
rev.relac.int.estrateg.segur.3(2):113-134,2008

\title{
RECONCEPTUALIZANDO LA SEGURIDAD: CAMBIO DE DILEMAS Y AMENAZAS
}

\author{
Mariana Delgado Barón*
}

\section{RESUMEN}

El artículo, presenta algunas de las transformaciones que ha sufrido la conceptualización de la seguridad, en cuanto a la definición del término y en cuanto a sus principales referentes, amenazas y dilemas. El proceso de reconceptualización de la seguridad plantea adicionalmente la redefinición del papel de los medios militares que tradicionalmente la garantizaban, y del principio de soberanía estatal. Dicha redefinición es también el resultado del cambio en la naturaleza de las amenazas y del surgimiento de otro tipo de actores diferentes al Estado que interactúan con éste en los ámbitos nacional e internacional. Por último, en el artículo se expone una breve caracterización del tipo de amenazas no tradicionales que han visibilizado otro tipo de problemas y asuntos referentes a la seguridad humana.

Palabras clave: Seguridad, seguridad humana, amenazas tradicionales y no tradicionales, migraciones, degradación medioambiental.

* Politóloga y Magíster en Ciencia Política Universidad de Los Andes, Magíster en Política Internacional Universidad de Birmingham, actualmente estudiante de Doctorado en Ciencias Sociales Facultad Latinoamericana de Ciencias Sociales- FLACSO, sede México. E-mail: marianadelgadob@yahoo.com. 


\begin{abstract}
The article presents some of the changes that the security concept has faced, specifically those related with the definition of the term, its main object, its threats and dilemmas. The reconceptualization process of security is also a redefinition of the role played by military mechanisms that traditionally have guaranteed it, and it also implies a redefinition of the sovereignty principle. This process of security reconfiguration is the result of the changes in the nature of the threats and also of the strengthening of different non- state actors that interact with the state. In the article there is also presented a brief characterization of some of the non- traditional threats related with new problems and issues.
\end{abstract}

Key words: Security, human security, traditional and non- traditional threats, migrations, environmental degradation.

\title{
INTRODUCCIÓN: «SOBRE LA CONCEPCIÓN Y CONCEPTUALIZACIÓN DE LA SEGURIDAD»
}

Hablar hoy en día, sobre el amplio concepto de seguridad supone dentro del imaginario común hacer referencia a la lucha contra el terrorismo y la configuración de las organizaciones criminales transnacionales, el uso del aparato militar, los mecanismos de cooperación interestatal, la idea e imagen del enemigo, el interés nacional y la subdivisión del mundo y de los Estados mismos entre «buenos» $y$ «malos», por mencionar solo algunos referentes. No obstante, estos referentes comunes sobre la seguridad han dado un giro, producto de la redefinición del concepto que ha implicado una visión más integral y compleja del mismo.

El concepto de seguridad a partir de los años noventa, supone que no sólo la posibilidad de guerra o conflicto a escala internacional constituye una amenaza para la integridad de los Estados, sino que factores internos que no se vinculan necesariamente con dinámicas de carácter militar representan serias amenazas no sólo para los Estados, sino también para las comunidades y los individuos.

En este sentido, el presente artículo tiene por objeto presentar el concepto de seguridad desde dos de sus dimensiones, que aunque no necesariamente son contradictorias, plantean problemáticas diferentes en los niveles internacional, estatal e individual sobre aquellos fenómenos o factores que repercuten directamente en la búsqueda u obtención de la seguridad de los Estados, las comunidades y los individuos. Asimismo, se parte del reconocimiento de la necesidad de ir más allá de la definición tradicional del concepto de seguridad, ya que ésta limita el análisis y la comprensión de realidades que constituyen amenazas a la seguridad en 
sus diferentes niveles como son las hambrunas, la pobreza que deriva en miseria extrema de numerosas poblaciones repartidas a lo largo y ancho del mundo, la tragedia medioambiental, las considerables dimensiones que han adquirido los flujos migratorios, y los conflictos internos, por mencionar algunas.

La vigencia del debate sobre la seguridad, radica precisamente en la importancia que continúa teniendo el concepto en términos explicativos y normativos de las acciones que han emprendido y que deben emprender los Estados y las mismas comunidades para garantizar determinados niveles de seguridad, que a su vez se requieren para obtener niveles mínimos de desarrollo. Además, la pertinencia de la discusión sobre el alcance y las dimensiones de la seguridad alude por otro lado a la necesidad de construir consensos mínimos frente a los elementos comunes que encierran los diferentes niveles del concepto.

«El estudio de la seguridad es central para el análisis de las relaciones internacionales» (Terriff, et al.; 2005:1), porque tradicionalmente ésta ha constituido el fin último del Estado, su principal objetivo y el más codiciado valor, a tal punto que los Estados están dispuestos a «competir» por obtenerla. Durante la guerra fría, la seguridad se enmarcaba en una perspectiva militarista que buscaba librar al Estado de cualquier ataque externo (y también del enemigo interno). No obstante, como se desarrolla más adelante, la seguridad ahora es un elemento más complejo, lo que ha impedido un consenso sobre su significado o definición.

Terriff, Croft y Morgan (2005:3), argumentan que la ausencia de una definición consensuada del término y las diversas aproximaciones teóricas al tema de la seguridad hacen que surja la pregunta sobre cómo definirla, pregunta que a su vez viene acompañada de variados cuestionamientos sobre quienes detentan la seguridad, qué valores se preservan, cuánta seguridad es necesaria, a través de qué medios, a qué costo, en qué periodo, y qué tipo de amenazas se combaten.

Contrario a esta complejidad que ha adquirido el término con sus nuevos desarrollos, la concepción tradicional ligada a una visión realista de las relaciones internacionales identifica la seguridad como un atributo exclusivo de los Estados, y de esta forma, el tipo de amenazas que se combaten aluden a la invasión del territorio y en términos más amplios a la defensa de la comunidad política que éste representa. La seguridad «realista» busca la preservación de una serie de valores comunes compartidos por la comunidad nacional; más específicamente, los Estados buscan conservar su independencia política y su integridad territorial (Baldwin; 1997:13).

Por tanto, la seguridad dentro del mundo realista está determinada por la capacidad de ejercer poder para garantizar la supervivencia en un sistema internacional regido por el principio de anarquía. «Todos los realistas sostienen que las manifestaciones y el impacto del poder y de la 
inseguridad es lo que le da a la política internacional una identidad distintiva» (Baldwin; 1997:39). Por lo tanto, el concepto de seguridad que se inscribe en la concepción realista de las relaciones internacionales, es también la capacidad de un Estado de detentar poder militar para someter a aquellos que cuentan con menos posibilidades de desarrollar su aparato armamentista.

Pero la concepción tradicional de la seguridad ha perdido relevancia para interpretar las actuales amenazas que enfrentan los Estados, dado el cambio de naturaleza de las mismas. Los planteamientos de la seguridad realista se quedan cortos al explicar las interacciones entre Estados y actores no estatales, al igual que al analizar las implicaciones hoy en día de las hambrunas, la pobreza y las migraciones, por señalar sólo algunas de las amenazas no tradicionales, en la seguridad de los Estados. Lo anterior no sólo porque éstos han dejado de ser los únicos actores o protagonistas dentro del sistema internacional, sino porque aspectos como los conflictos internos, la pobreza, el desempeño económico de los países y las hambrunas se han convertido en desafíos considerables que los Estados y los mismos individuos deben enfrentar para preservar su seguridad. De este modo, los individuos, al igual que los Estados, buscan preservar una serie de valores para sentirse "seguros», como la autonomía y el bienestar económico (Baldwin; 1997:39).

Aquello que determina la conceptualización de la seguridad y que se convierte en el elemento diferenciador de las distintas aproximaciones al término, es principalmente su «objeto de referencia» y por consiguiente la naturaleza de sus amenazas. Tradicionalmente, el referente de la seguridad lo constituía el Estado, por tanto la amenaza a la seguridad estatal se percibía en términos de violación a su soberanía, siendo los medios militares el mecanismo más apropiado para garantizarla.

A partir de la década de los noventa, se viene consolidando una aproximación diferente de la seguridad en cuanto a su punto de referencia, los medios para alcanzarla y el tipo o naturaleza de las amenazas. De este modo, la transición de una concepción de la seguridad «estadocéntrica» hacia una concepción que concibe a las comunidades y más particularmente al individuo como referencia, centra su atención en los mecanismos que «liberan a la gente de los constreñimientos o restricciones físicas, sociales, culturales que impiden hacer aquello que cada individuo haría libremente». Las hambrunas, las enfermedades, las catástrofes naturales, las nuevas guerras, el terrorismo y el desempleo son algunas de las amenazas que enfrenta el proceso de consolidación de una seguridad dirigida a garantizar las condiciones para la emancipación del hombre. En otras palabras, el concepto de seguridad implica garantizar no sólo la integridad territorial del Estado, sino también velar por otros valores como la libertad, la justicia, la equidad, la distribución de la riqueza, por nombrar algunos. 


\section{Nuevos desarrollos.}

Con el fin de la guerra fría se han venido consolidando nuevas perspectivas y aproximaciones al concepto de seguridad. Estas transformaciones han traído consigo un cambio de dimensiones que abarcan otro tipo de amenazas no tradicionales, haciendo que la seguridad sea un concepto más incluyente en cuanto a sus dimensiones, dilemas, dinámicas y amenazas.

El fin de la confrontación ideológica entre comunismo y capitalismo, ha implicado en cierta forma la necesidad de replantear la concepción tradicional de la seguridad, inscrita en el marco realista de las relaciones internacionales; hecho que ha incidido en el surgimiento de nuevas discusiones sobre quién o qué constituye el enemigo, y sobre el papel que juegan los factores domésticos en los asuntos de seguridad (Baldwin; 1997:4). Como producto de la finalización del enfrentamiento bipolar, han surgido discusiones acerca de la función que debería cumplir el poder militar, y cuestionamientos sobre cómo analizar la forma en que se piensa y se concibe la seguridad, asuntos que derivan en la ampliación de dicho término (Zinder; 1999:7). Tradicionalmente, el concepto de seguridad ha estado relacionado con la capacidad de un Estado para cuidar sus fronteras nacionales de amenazas externas o guerras interestatales; pero hoy en día, la noción de inseguridad es el resultado de preocupaciones sobre la vida diaria.

La reconceptualización de la seguridad implica entonces prestar atención a cómo los conflictos ambientales, la lucha por los recursos naturales, las migraciones, el terrorismo, las organizaciones criminales transnacionales, la misma privatización de la seguridad, las pandemias, las hambrunas, el desempleo, la proliferación de estados fallidos (failed states), las nuevas guerras y los estados canallas (rogue states), entre otros, se conciben como amenazas y desafíos para su obtención y consolidación.

Estas transformaciones conceptuales han generado un «nuevo y fortalecido significado [del término, que a su vez ha consolidado] una «nueva era» de seguridad» (Moreno; 2005:150). Esto quiere decir que aparte de nuevas aproximaciones, se ha dado un cambio frente al monopolio de la fuerza ejercido por el Estado, en cuanto a los tipos de actores no estatales que compiten entre sí por este recurso en el ámbito nacional e internacional, como los grupos al margen de la ley o los grupos criminales transnacionales. En este sentido, la visión tradicional de la seguridad (nacional), que se encuentra atada a la teoría realista, defiende al Estado como único detentador del monopolio de la fuerza que tiene como fin principal la preservación de un territorio y la defensa de una comunidad nacional, y el principal objeto de la seguridad. Para los realistas, no existe ningún otro actor al interior del territorio nacional que pueda competir con el Estado por el monopolio del uso de la fuerza; sin embargo, en el ámbito internacional y debido a la anarquía del sistema, se presenta un «estado de naturaleza» de todos contra todos en el que se lucha por el poder. 
La seguridad, según los realistas, se reduce a la preservación de la integridad del territorio nacional frente a las amenazas que puedan socavarla, como puede serlo la invasión de un país enemigo. Bajo los planteamientos realistas, las amenazas que enfrentan los Estados son «fenómenos identificables que pueden ser controlados por los líderes nacionales, debido a que son realidades que pueden modificarse por medio de actos deliberados de implementación de decisiones políticas tendientes a contrarrestarlos» (Moreno; 2005:154).

La preservación de la seguridad bajo la óptica realista se explica también con el conocido «dilema de la seguridad», que especialmente fue utilizado durante la guerra fría para analizar el enfrentamiento bipolar. Dicho dilema permitió establecer los escenarios de enfrentamiento y conflicto y la probabilidad de que estas situaciones de guerra surgieran entre los países. A su vez, el dilema plantea cómo las acciones adoptadas por un Estado para salvaguardar su seguridad son interpretadas por otro como una amenaza a sus intereses nacionales; "en el entorno conflictivo de la mencionada anarquía internacional, la ocurrencia de una guerra es más probable cuando los medios a través de los cuales un Estado intenta aumentar su seguridad, ocasionan o tienen como efecto el decrecimiento de la seguridad de los demás Estados» (Moreno; 2005:156).

La aproximación realista a la seguridad, reflejada en plena guerra fría, a través del dilema de la seguridad y de sus planteamientos básicos enfoca el problema hacia un asunto estatal y militar, en otras palabras, la «seguridad realista» es un concepto convencional que se inscribe en una visión estado-céntrica de la política nacional e internacional.

Sin embargo, el cambio de un sistema bipolar a uno multipolar (o uni-multipolar en palabras de Samuel Huntington) en el que se han visibilizado problemas que la confrontación ideológica Este-Oeste había permeado, hace necesaria la construcción de una aproximación diferente a la seguridad. La redefinición del concepto ha sido un fenómeno producto de otros hechos y factores ligados al fin de la polarización ideológica como son «los cambios en la práctica de la guerra moderna (...), la ruptura entre la necesidad de contar con seguridad para el entorno propio y la preocupación moral por la seguridad de los otros (...), las nuevas formas de relación entre los Estados democráticos, (... y la vinculación entre la defensa del Estado, en abstracto, y la garantía de las necesidades humanas básicas en concreto» (Aguirre; 2000:6).

El panorama que viene constituyendo las relaciones entre Estados y en el que han surgido nuevos tipos de actores, protagonistas también de las relaciones internacionales, es el espejo de un ordenamiento mundial caracterizado por la búsqueda permanente de la paz internacional y de una seguridad dirigida no sólo a evitar los conflictos interestatales, sino también a conseguir la implementación de Democracias liberales alrededor del mundo.

Como bien lo señala David Baldwin, la redefinición del concepto de seguridad ha estado ligada con una redefinición de las agendas políticas de los Estados, en las que se ha prestado mayor 
atención a los derechos humanos, la economía, el medio ambiente, el tráfico de drogas, el crimen, las epidemias y la justicia social (1997:5).

Autores como Terriff, Croft y Morgan (2005:3) señalan que la nueva definición de la seguridad debe incluir varios niveles de análisis que se complementan unos con otros como son el nivel de los Estados, el individual, el familiar y el social, contrario a los planteamientos de otros teóricos como Barry Buzan, que resaltan la inconveniencia de analizar el concepto de seguridad bajo diferentes instancias, ya que éste es uno solo y por tanto debe ser entendido y analizado en su totalidad.

A lo anterior se suman las discusiones que surgen a raíz de la ampliación del concepto de seguridad, en la medida en que se plantea que «no todo tema importante es un tema de seguridad, y tampoco todo tema de seguridad es per se un tema importante» (Rojas; 2000:3). La ampliación conceptual ha llevado a que el concepto incluya un poco de todo y no particularice en un asunto específico, lo que para algunos ha producido una deformación del término.

Autores como Buzan y Ullman categorizan los asuntos de seguridad como aquellos que requieren de una acción rápida o urgente. Para ejemplificar, Ullman señala que los asuntos o problemas que se consideran de seguridad, son aquellos «que degradan la calidad de vida de la población de un Estado de forma drástica y rápida», en este sentido, declarar que algo es un asunto de seguridad es validar su importancia (Baldwin; 1997:20).

Frente a este debate, se ha señalado «el desafío intelectual» que nace de la urgencia de poder y de saber «ligar y establecer una concatenación conceptual desde la seguridad humana hacia la seguridad internacional pasando por la seguridad estatal»(Baldwin; 1997:5), planteamiento que sugiere y refuerza la idea sobre las diversas escalas o niveles bajo los cuales se debe definir, analizar y comprender la seguridad.

\section{LA SEGURIDAD HUMANA: EL HOMBRE COMO PUNTO DE REFERENCIA}

Reconceptualizar la seguridad y dirigir su significado hacia la condición de plena garantía de las necesidades básicas de los individuos como la alimentación, la vivienda, la salud, la educación, el trabajo, implica a su vez el replanteamiento del uso de la fuerza y su finalidad última. El uso de la fuerza para defender un territorio, o el uso de la fuerza para preservar el orden interno y en general para resolver los problemas intraestatales, son dos cuestiones que se sitúan en el centro del debate sobre qué elementos y qué factores determinan y constituyen la seguridad.

El concepto de seguridad humana encierra una definición mucho más amplia del término y está encaminada a otros referentes. Durante décadas, teóricos de las relaciones internacionales 
consideraban la seguridad como un atributo exclusivo de los Estados, que adquiría sentido por medio de la permanente posibilidad de conflicto entre éstos. «Por mucho tiempo la seguridad ha sido equiparada [con la protección del territorio nacional de cualquier amenaza]; por mucho tiempo, las naciones han visto como las armas protegen [y garantizan] su seguridad» (Mesajasz; 2004:5). A pesar del auge que tuvo el concepto de la «seguridad realista» para entender y analizar varias de las situaciones durante la guerra fría, el concepto de seguridad últimamente ha tendido a centrarse más en los individuos y no en los Estados, dando paso a una seguridad a través del desarrollo humano (PNUD:1993).

La seguridad humana, implica estar a salvo de las constantes amenazas de hambre, enfermedad, crimen, represión, drogas; implica que los individuos se sienten protegidos de violentas o repentinas rupturas en su vida diaria, "la seguridad humana se concentra en sobrevivir, en la vida diaria y en la dignidad de los seres humanos» (Sen: 2000).

El concepto de seguridad humana hace referencia a la «seguridad interna de los ciudadanos» en la que se destaca el papel que la protección de los derechos humanos, la igualdad de oportunidades frente al acceso de bienes esenciales, la coexistencia armónica entre el hombre y el medio ambiente y otro tipo de condiciones juegan dentro una verdadera implementación y conservación de un estatus de seguridad.

La seguridad humana constituye la liberación de los individuos del miedo y de la necesidad, razón que obliga a prestar atención a las condiciones que aseguran los derechos económicos, políticos, culturales y físicos de la población (Der Derian: 2005). El concepto de seguridad humana se subdivide así en seis categorías según el tipo de amenazas que pueden afectar la vida diaria de los individuos: la económica, la alimenticia, la medioambiental, la personal, la comunitaria y la política (PNUD: 1994).

Las pandemias, los conflictos étnico- religiosos, la degradación del medio ambiente, las migraciones masivas y la descomunal brecha entre ricos y pobres son fenómenos que se han agravado con el fin de la guerra fría. «El mundo de la posguerra fría es más inestable y menos predecible; las amenazas que enfrentamos son más difusas y multidimensionales. En muchas formas, el mundo es menos seguro hoy, particularmente para los civiles» (McRae; 2001:15).

En consecuencia, la ampliación del concepto de seguridad traduce el impacto que amenazas no tradicionales han provocado en la estabilidad del orden internacional y en la preservación de la soberanía y ordenamiento interno de los Estados, especialmente cuando la «sensación de inseguridad»s se relaciona no tanto con la posibilidad de una guerra interestatal, sino con la incertidumbre del hombre frente al futuro del medio ambiente, la economía, el empleo y demás (Aguirre; 2000:6). El sistema internacional predominante durante el siglo XX fue configurado de 
tal forma que se diera un predominio a la seguridad estatal y por ende a la soberanía de los Estados, y no tanto a la protección de los civiles al interior de los mismos (McRae; 2001:15). «En el periodo de posguerra fría ha aparecido con fuerza la dimensión de la seguridad humana como un concepto articulador clave en un mundo en transición» (Rojas; 2000:4).

El concepto de seguridad humana es muy amplio, tanto que en ocasiones se confunde con el desarrollo humano y otros instrumentos para su promoción como los derechos humanos y el derecho internacional humanitario. Sin embargo, el concepto y la aplicación de la seguridad humana trascienden la esfera normativa de los DD.HH y del $\mathrm{DIH}$, ya que principalmente busca que los individuos estén a salvo de cualquier tipo de violencia que amenace una calidad óptima de vida.

Cabe mencionar que garantizar la seguridad humana es un gran desafío para aquellos Estados que carecen de los mecanismos necesarios para implementar acuerdos internacionales, ya sea porque enfrentan graves problemas de gobernabilidad, corrupción, o conflictos étnicos, considerables flujos de desplazados y refugiados, hambrunas y enfermedades contagiosas, entre otras problemáticas. En estos casos de Estados colapsados, la comunidad internacional se ve en la necesidad de actuar, tiene la «responsabilidad de proteger», bajo la figura de la intervención humanitaria, a aquellos ciudadanos cuyas necesidades humanas básicas no han sido aún garantizadas. De esta forma, los instrumentos o mecanismos para garantizar la seguridad humana requieren de la cooperación entre los Estados, hecho que puede limitar la soberanía de los mismos Así mismo, «los acuerdos intergubernamentales en el área de la seguridad humana internacionalizan los asuntos de la seguridad que antes pertenecían al dominio de la política interna»(McRae; 2001:25).

Por otro lado, el concepto de seguridad humana tiende a ser asociado con una «seguridad blanda» (soft security) ligada a los asuntos de la «baja política», donde el uso de la fuerza no es el mejor mecanismo para afrontar y dar respuesta a las problemáticas no tradicionales que predominan dentro de esta concepción. La «seguridad dura» (hard security), como competencia de la «alta política» se vincula con el significado tradicional del término, y por tanto sitúa en el centro de acción a las fuerzas militares que continúan desempeñando una función predominante para enfrentar las amenazas de carácter tradicional (Liotta; 2005).

Frente a la existencia de dos «tipologías» de la seguridad es importante reconocer sus diferencias. La seguridad dura se asocia directamente con la fuerza militar, que busca proteger los intereses nacionales y defender al Estado contra amenazas de agresión externa. Las amenazas que se combaten por medio de la implantación de esta seguridad, son claramente visibles y comúnmente identificables. 
El concepto de seguridad blanda relaciona amenazas con «vulnerabilidades», que son percibidas como indicadores, no siempre claramente identificables y generalmente vinculados a una serie de problemáticas complejas que deben ser abordadas por medios diferentes a los militares.

A pesar de la diferenciación y caracterización de estos dos tipos de seguridad, ésta sigue concibiéndose como una totalidad dirigida a enfrentar y dar respuesta a diferentes tipos de amenazas que van desde el narcotráfico y las organizaciones criminales, hasta el desempleo, el conflicto social, las hambrunas y los bajos niveles de representación política.

Así mismo, la seguridad humana se acerca a la eliminación o por lo menos a dar un manejo a las situaciones y condiciones de extrema vulnerabilidad bajo las que viven amplios sectores sociales marginados de los procesos de desarrollo. El logro principal de la seguridad humana es asegurar un desarrollo humano de corto, mediano y largo plazo, «la seguridad humana requiere que la gente viva en dignidad y paz, que las personas tengan suficiente para comer, que puedan encontrar trabajo, y que vivan en un mundo sin pobreza» (Bajpai; 2000:7). Siguiendo esta misma concepción, las Naciones Unidas, bajo su Programa de Desarrollo, ha señalado que la seguridad significa para muchos «protección de las amenazas de enfermedad, hambre, desempleo, crimen, conflicto social, represión política, peligros medioambientales (...) [en otras palabras], la seguridad humana es un niño que no muere, una enfermedad que no se expande, un trabajo que no se elimina, una tensión étnica que no explota en violencia, un disidente que no es silenciado» (Bajpai; 2000:14).

Según lo anterior, El Programa de Desarrollo de Naciones Unidas aclara que la seguridad humana, más que ser un asunto de armas está directamente relacionado con la dignidad y la vida humana, particularmente es un asunto que se vincula con los componentes de la seguridad económica, alimentaria, de la salud, medioambiental, comunitaria, política y personal (Bajpai; 2000:14).»La seguridad humana es una condición de existencia en las que las necesidades materiales se realizan, y en la que la dignidad humana es posible» (Alkire; 2003:15).

La seguridad, se ha convertido en una cuestión que no sólo concierne con la preservación de las fronteras del Estado, sino que sobretodo se relaciona con garantizar a la población el pleno cumplimiento de sus libertades y derechos.

El tipo de amenazas a la seguridad humana, se extiende en un abanico que comprende desde la ausencia o escasez de empleo remunerado y el subdesarrollo económico hasta el genocidio y las violaciones sistemáticas a los derechos humanos. Por lo tanto, los principales valores que persigue la seguridad humana son la protección del individuo del dolor y la destrucción, las libertades básicas que posibiliten la escogencia de un estilo de vida, y la libertad cívica que permita a los individuos la libertad de organización (Bajpai; 2000:37). 
Para varios autores el debate sobre la seguridad, se centra entonces en determinar qué pesa más en la balanza o qué es más importante: si la seguridad orientada a salvaguardar la integridad del territorio nacional a partir de medios militares o la seguridad que se dirige a garantizar la integridad de los individuos al interior de un territorio por medio de estrategias que impulsen el desarrollo humano.

\section{TRANSFORMACIÓN EN LA NATURALEZA DE LAS AMENAZAS: ¿CONTRA QUÉ SE COMBATE?}

Como bien se señaló en párrafos anteriores, el ambiente de la seguridad global se ha modificado considerablemente, especialmente en lo referente al carácter de las amenazas que afectan la estabilidad del orden internacional. A pesar de las transformaciones en la naturaleza de las amenazas a la seguridad, los Estados continúan haciendo uso de la fuerza, del poder militar, para alcanzar sus objetivos y proteger sus intereses nacionales. No obstante, los mismos Estados perciben de una forma diferente los problemas que pueden afectar su estabilidad. La concepción tradicional de la seguridad se refiere a «cómo los estados usan la fuerza para enfrentar o evitar amenazas a su integridad territorial, a su autonomía y a su ordenamiento político nacional, que provienen de otros estados» (Bajpai; 2000:3). No obstante, limitar los asuntos de seguridad a cuestiones de carácter militar, implica dejar de lado problemáticas como la pobreza, las hambrunas, la degradación del medio ambiente, el desempleo, entre otras, que afectan la seguridad de los individuos y de las comunidades. De cierta forma, lo anterior supone que «existen amenazas a la seguridad humana que van más allá de las capacidades del Estado para enfrentarlas» (Bajpai; 2000:37).

Asuntos como los conflictos internos, la proliferación de armas de destrucción masiva, la estabilidad y la cooperación regional, y el control de armas, continúan siendo una preocupación de los Estados y de la comunidad internacional en general; sin embargo, a estas preocupaciones se suman otro tipo de amenazas referentes a la degradación del medio ambiente, las migraciones, el terrorismo, el desempeño económico de los países, las organizaciones criminales transnacionales y las mismas dimensiones que pueden adoptar las guerras internas en cuanto a la estabilidad política, social y económica de los Estados.

Estas amenazas no tradicionales a la seguridad a pesar de ser diferentes y de centrarse en asuntos particulares, comparten una serie de características a saber: a) no se dirigen exclusivamente al Estado; b) no se sitúan en un área geográfica determinada y específica, son amenazas difusas; c) no pueden ser manejadas a través de métodos militares únicamente; otros medios de carácter no militar deben ser empleados; d) los individuos y las comunidades, al igual que los Estados, son objeto de la seguridad, y por ende pueden estar en peligro (Terriff; 2005:116). 
Es importante señalar que mientras algunos autores resaltan que el uso de la fuerza es sólo uno de los varios instrumentos que pueden ser empleados para garantizar la seguridad humana, lo cuestionable o dudoso es si es el medio más efectivo (McRae; 2001:25), otros mencionan que las políticas que se toman en las cuestiones de seguridad deben ser más inclusivas, de tal forma que se encuentren integradas más ampliamente con estrategias para la promoción de los derechos humanos, la democracia y el desarrollo (Bajpai; 2000:32). "La defensa de la nación contra enfermedades contagiosas es un problema completamente diferente que la defensa contra el ataque de un misil balístico. La primera situación corresponde a una amenaza cuya respuesta requiere reforzar la cooperación internacional y buscar formas de prevención más vinculadas a la salud y al desarrollo. La segunda situación está ligada directamente a los problemas de defensa y seguridad» (Rojas; 2000:5).

También se encuentran aproximaciones a la seguridad en las que se afirma que para garantizar la seguridad internacional se debe prestar mayor atención a la dimensión humana de este concepto, esto especialmente en aquellos países que enfrentan graves crisis humanitarias, en donde se requiere en gran medida de asistencia internacional para garantizar las necesidades humanas básicas. Continuando con esta idea, según la visión canadiense de la seguridad humana, la seguridad de los individuos es una forma de medir la seguridad global, en otras palabras, "la seguridad de los Estados es una condición necesaria para la seguridad de la gente» (Bajpai; 2000:17), la segunda no obvia la primera.

A continuación se señalan algunas de las principales características de las amenazas no tradicionales a la seguridad que representan grandes desafíos para la supervivencia de los individuos, las relaciones entre los estados, y la configuración misma del orden internacional.

\section{La degradación medioambiental}

Los problemas derivados de la degradación del medio ambiente no surgieron de repente con el fin de la guerra fría, se han venido presentando desde que el crecimiento de las economías industrializadas depende directamente de la explotación de los recursos naturales.

Thomas Homer-Dixon (1994) ha analizado la incidencia de la escasez de los recursos naturales en los conflictos internos, particularmente como un asunto de los países en vías de desarrollo. Para Homer-Dixon este tipo de fenómenos afectan a países pobres, debido a que sus gobiernos no disponen de las capacidades necesarias para controlar las crisis sociales que derivan del mal uso de los recursos naturales.

Para el autor, la degradación del medio ambiente puede producir en estos países tres tipos diferentes de conflictos que afectan directamente la seguridad intraestatal y humana con serias repercusiones en la seguridad interestatal. Por un lado, el decrecimiento de las reservas de 
recursos naturales como el agua y la tierra cultivable provocarán guerras o conflictos entre la población que busca un acceso a los mismos. Adicionalmente, los movimientos migratorios causados por la escasez de los recursos naturales producirán conflictos étnicos entre comunidades con identidades culturales bien definidas. Por último, la degradación medioambiental incrementará el estancamiento económico de estas sociedades, y a su vez promoverá la crisis de las instituciones sociales, la agudización del crimen organizado y movimientos de insurgencia armada.

Autores como Terriff, Croft y Morgan, han señalado que diferentes nociones sobre seguridad medioambiental defienden la idea sobre cómo la degradación del medioambiente representa una amenaza significativa a los individuos, las comunidades y a los mismos Estados. Dichas nociones pueden ser agrupadas en tres categorías diferentes:

- $\quad$ La degradación del medio ambiente es una causa de inestabilidad política y de conflicto. - $\quad$ La degradación del medio ambiente puede ser producto de la guerra.

- $\quad$ La degradación del medio ambiente representa una amenaza a la salud humana y a la calidad de vida de los individuos.

A pesar de los riesgos que la escasez de los recursos naturales generan para la seguridad humana, autores como Daniel Deudney (2005) argumentan que no existe vínculo alguno entre degradación ambiental y seguridad nacional, básicamente porque la primera no representa ninguna conexión con la protección estatal de la violencia ejercida por otros Estados. De esta forma, para Deudney, la degradación medioambiental no constituye ninguna amenaza a la seguridad de los Estados. Aún así, Deudney plantea 5 formas diferentes en las que la degradación del medio ambiente podría producir «teóricamente» conflictos entre los estados que impliquen violencia organizada: a) Guerras por el control de los recursos naturales no renovables; b) La degradación medioambiental puede generar inestabilidad interna que podría producir violencia interestatal; c) La degradación del medio ambiente puede alterar materialmente el poder relativo de los Estados, lo que podría generar conflictos entre éstos; d) La polución expandida más allá de las fronteras estatales puede causar conflictos entre los Estados; y e) Problemáticas comunes como el cambio climático y el aumento del deterioro de la capa de ozono podrían producir guerras y conflictos entre los estados (2005:3).

Cabe resaltar que dichas críticas se inscriben en una concepción realista de la seguridad, pues defienden el significado tradicional del término que se centra en la seguridad nacional, orientada a impedir la violencia organizada ejercida por otro Estado. Bajo esta aproximación tradicional, si la degradación del medio ambiente y otro tipo de amenazas no tradicionales como las enfermedades infecciosas e incluso el tráfico de drogas no están conectados de alguna forma con violencia organizada interestatal, estos problemas no pueden ser considerados asuntos de seguridad nacional (Deudney; 2005:5). 
Sin embargo, es visible el aumento de los desafíos ambientales que acompañan el desarrollo y la consolidación de las economías industrializadas, que a su vez dependen de la explotación de los recursos ambientales. Además, el acceso a determinados recursos naturales como el petróleo, puede considerarse un factor importante para el afianzamiento del poder estatal, que a su vez garantiza una mayor seguridad y estabilidad.

Considerar que la degradación del medio ambiente es un factor que genera inseguridad en diferentes niveles implica aceptar la redefinición del concepto, especialmente en lo referente a su objeto; la seguridad no sólo afecta los Estados, sino que se extiende también a los individuos, grupos, regiones y bio-regiones (Terriff; 2005)

\section{Desempeño económico y seguridad}

Para muchos Estados en el mundo, las amenazas no tradicionales a la seguridad son percibidas como asuntos más importantes y «peligrosos» que aquellos que se encuentran representados por el poder militar detentado por otros Estados.

Al igual que la degradación del medio ambiente, el desempeño económico es una amenaza no tradicional que juega un importante papel frente a la reconceptualización de la seguridad y específicamente frente a los desafíos que impone a la misma. A partir de la década de los 90 se comenzó a gestar la idea de una nueva era mundial en la que los aspectos económicos de la seguridad dominarían las dimensiones militares y políticas de las relaciones entre los Estados y al interior de los mismos. El protagonismo adquirido por los factores económicos en el ordenamiento internacional y en el conjunto de elementos indispensables para preservar la paz y la estabilidad mundial, se hace visible a través del cálculo que realizan los Estados para localizar sus limitados recursos entre obtener seguridad en el corto plazo y asegurar un bienestar económico en el largo plazo. En este punto es fundamental mencionar que a pesar de considerar el crecimiento económico como un asunto de seguridad en el sentido no tradicional, la principal referencia de los análisis sobre la seguridad económica continúa siendo el Estado, aún cuando ésta se considera un componente de la seguridad humana, ya que los individuos permanentemente buscan conseguirla.

Los vínculos entre seguridad y desempeño económico se subdividen en tres categorías: a) El potencial económico se relaciona con el poder, con la capacidad de un Estado de ejercer fuerza e influencia; b) el buen desempeño económico del Estado hace parte de sus valores primarios, y c) los medios económicos son utilizados por los Estados para alcanzar sus fines (Terriff; 2005:137).

Cada una de estas categorías refuerza la idea de una "seguridad económica» en la que los medios económicos y materiales son valores esenciales de los Estados que deben ser protegidos. 
En el caso de países con economías pobres o deterioradas esta situación puede afectar su estabilidad política y social, y por tanto su seguridad interna; además, una posición económica débil puede conducir a imposiciones extranjeras sobre la soberanía nacional. Así mismo, la búsqueda de la consolidación económica por parte de los países en vías de desarrollo, es también un proceso que incluye el fortalecimiento y la consolidación del Estado como una nación alrededor de un consenso político y social. Tal proceso de consolidación de la nación hace aún más difícil y lenta la labor de consolidar la economía nacional y viceversa, una economía débil restringe la capacidad de acción y de manejo de las demandas sociales por parte de los gobiernos, alimentando las divisiones y los conflictos internos.

Aquellos Estados con economías deterioradas enfrentan problemas de tres tipos: a) la ausencia de crecimiento económico restringe la capacidad de los Estados para dar respuesta a las demandas que surgen desde la sociedad; b) Estados con economías estancadas juegan un papel mínimo en el sistema internacional, y c) los Estados con economías debilitadas son altamente propensos a recibir influencias extranjeras, hecho que puede provocar divisiones internas y por ende fragmentación social (Terriff; 2005:142).

Del mismo modo, los Estados buscan obtener «seguridad económica» para garantizar no sólo mínimos de cohesión social, sino también para ir configurando un proyecto conjunto de nación.

«Los instrumentos económicos son importantes herramientas que los Estados emplean para proyectar sus intereses nacionales»(Terriff; 2005:142), el poder económico que surge a partir de un considerable crecimiento y desarrollo económico posibilita a los Estados que lo experimentan jugar un papel preponderante en las decisiones que deben adoptarse en el ordenamiento internacional. Incluso, algunos Estados han optado por el uso de instrumentos de naturaleza económica con un fin punitivo, por ejemplo cuando emplean sanciones económicas como medio de presión y así coactar a otros Estados para que se comporten de una manera en particular o para que adopten determinadas medidas.

Esto permite señalar que los Estados con economías fuertes y posicionadas detentan mayor influencia en el escenario internacional, mientras que los Estados con economías debilitadas, son altamente susceptibles de ser presionados por los primeros, circunstancia que implica pérdida de autonomía. En este mismo sentido, existe una gran conexión entre «riqueza» y poder militar, ya que una economía fuerte provee los medios tanto materiales como financieros para el desarrollo de la capacidad militar (Terriff; 2005:137).

Por otro lado, la seguridad económica al nivel de los individuos, se convierte en un objetivo o interés a perseguir, ya que ésta garantiza la realización o concreción material de una forma de vida que se ha escogido libremente. La seguridad económica posibilita hacer realidad las 
aspiraciones materiales de los individuos, al igual que puede evitar en ellos sentimientos de frustración que se generan a partir del desempleo provocado por crisis económicas o estancamiento del sistema.

\section{Organizaciones criminales transnacionales: el terrorismo a gran escala}

La existencia dentro del sistema internacional de actores diferentes a los Estados, con capacidad de alterar el orden mundial, permite señalar que a pesar que las relaciones entre Estados se rigen por el principio de soberanía, los actores transnacionales, y en este caso, las organizaciones criminales, que no cuentan con un componente soberano, interactúan con los estados y pueden competir con éstos por el uso de la fuerza, a la vez que constituyen un desafío a la seguridad del estado, de las comunidades y de los individuos.

«Los [Estados], aún operan y aún son soberanos en numerosas formas (...), pero algo de su autoridad ha sido relocalizada en entidades o colectividades de carácter subnacional» (Roseneau; 1993:3) e incluso en entes de tipo transnacional.

Las organizaciones criminales transnacionales mediante el uso de la fuerza se enfrentan con los estados, socavando su legitimidad y compitiendo también por el control de territorios y el acceso a determinados recursos. El crecimiento de este tipo de organizaciones en cuanto a poder y capacidad de acción se ha extendido hacia formas de violencia política, infiltrándose en sectores políticos y sociales para proteger sus objetivos. La influencia de estas organizaciones no se limita a las esferas estatales, sino que se expande al ámbito interestatal, amenazando la estabilidad del sistema internacional. "[las organizaciones criminales transnacionales se convierten] en un desafío para la integridad del sistema de fronteras nacionales y para la habilidad de los estados de proteger a sus ciudadanos de la corrupción, de las organizaciones armadas ilegales y del tráfico de drogas» (Der Derian; 2005).

El crimen transnacional organizado financia sus actividades generalmente a través del tráfico de drogas y armas e involucra acciones ilícitas como la trata de niños y mujeres, el comercio de órganos humanos, y el financiamiento de conflictos internos a través del entrenamiento y la dotación de armamento a facciones armadas insurgentes en Estados débiles o fallidos, entre otros ejemplos.

Las organizaciones criminales transnacionales son variadas en términos de sus actividades, tácticas, estructuras y tamaños, «no existe un único modelo de organización criminal transnacional». No obstante, este tipo de organizaciones comparten o tiene en común como el uso de la violencia y de mecanismos de corrupción para defender sus intereses (Williams; 1999:175). 
La capacidad de provocar temor generalizado y de producir un daño intensivo en la población es el resultado del vínculo entre el crimen transnacional organizado y grupos terroristas que tienen «la capacidad y la voluntad de causar destrucciones y muertes masivas» (Williams; 1999:175). Después del 11 de septiembre, el terrorismo ha tomado un lugar central dentro del discurso sobre la seguridad internacional y se ha convertido en la causa principal de la redefinición de la estrategia de seguridad del sistema internacional.

El terrorismo ha seguido el mismo patrón de funcionamiento de las corporaciones multinacionales, aprovechando los procesos de globalización para la distribución de funciones y recursos, y alimentando su capacidad de acción en cualquier lugar del planeta. La estructura de funcionamiento del terrorismo transnacional y sus estrechos vínculos con otros tipos de organizaciones dificultan la labor de desmonte de los movimientos insurgentes que se valen de medios terroristas como fórmula de acción.

Los procesos de globalización han facilitado los lazos de interdependencia entre los Estados y han permitido concebir la idea de una sociedad civil global, pero así mismo han proporcionado los mecanismos para que las organizaciones criminales transnacionales se conviertan en una amenaza no sólo de tipo nacional e internacional, sino también humana. La globalización ha proveído importantes oportunidades para su acción y fines a las organizaciones criminales transnacionales, como la facilidad de movilidad a lo largo de las fronteras, el constante aumento del comercio internacional que posibilita la «conversión» de productos ilícitos en lícitos y las infraestructuras informáticas que facilita a este tipo de organizaciones realizar nuevas formas de extorsión, entre otras (Williams; 1999:180).

«Si las organizaciones criminales transnacionales pueden ser identificadas como la contraparte ilícita de las corporaciones transnacionales en su búsqueda de ganancia, también pueden tener muchas de las características de las asociaciones profesionales transnacionales», como el basar su organización en una estructura de redes (Williams; 1999:174).

A pesar de que es claro que las organizaciones criminales transnacionales se han convertido en una seria amenaza para los Estados y también para los individuos, éstas no constituyen un desafío en términos militares o no se conciben tanto como amenazas de carácter militar

\section{Migraciones internacionales: ¿amenaza a la integración social y a la identidad cultural?}

¿En qué sentido las migraciones pueden ser consideradas como amenaza a la seguridad de la comunidad internacional? ¿Constituyen las migraciones una amenaza a la integridad del Estado únicamente? ¿Representan las migraciones alguna amenaza a las comunidades?, estas son algunas de las preguntas que surgen alrededor del tema y que plantean a su vez el debate sobre la 
pertinencia y las implicaciones que se desprenden de la inclusión de este asunto dentro de la agenda de la seguridad.

Las migraciones generalmente han sido analizadas bajo los parámetros que delimita el Estado, en cuanto a que son consideradas como un peligro a la «homogeneidad nacional» y por tanto a la integridad misma de los Estados como unidades políticas. La figura del migrante se asocia con la de un invasor que amenaza la identidad nacional, la homogeneidad del Estado, de la sociedad y de incluso la política (Didier; 2002).

El debate sobre las migraciones como amenaza a la seguridad invita a repensar sobre el objeto y el tipo de valores que ésta última defiende. En este caso, los componentes de la seguridad que se ven alterados por las migraciones varían desde la estabilidad e integridad social, la identidad cultural, la seguridad interna y la seguridad demográfica (Kissinger; 2004:2). La estabilidad social puede ponerse en riesgo cuando los flujos de migrantes generan en las comunidades receptoras actos de xenofobia que dificultan más los procesos de integración. Del mismo modo, la migración internacional puede convertirse en una amenaza al sistema de bienestar y seguridad social cuando los migrantes son «económicamente inactivos» y viven de los beneficios sociales que le brinda el Estado receptor. Por último, en algunos casos, la migración internacional representa un riesgo para la seguridad interna cuando existe conexión entre los migrantes y el crimen organizado. Frente a esto último, las organizaciones criminales transnacionales «hacen uso tanto de la migración legal como ilegal para penetrar al país» (Kissinger; 2004:3).

«La migración internacional puede ser el producto de otro tipo de amenazas como (...) los conflictos internos y la guerra étnica; [por otro lado], puede constituir por sí misma una amenaza a la seguridad internacional cuando es masiva y no controlada, y puede desembocar en otras amenazas a la seguridad (xenofobia, violencia racial)» (Kissinger; 2004:3). En esta misma dirección, autores como Meilaender caracterizan la migración como un fenómeno que puede ser voluntario o involuntario, siendo este último producto de guerras o conflictos, desastres naturales, hambrunas, o tráfico de personas (Meilaender; 2001).

Los altos movimientos migratorios y el constante aumento de refugiados son una preocupación de los Estados no sólo por enfrentar situaciones de asistencia humanitaria en determinadas circunstancias, sino por que estos flujos de población representan para los gobiernos serios desafíos a su estabilidad social, cultural, política y económica. Del mismo modo, la migración irregular es concebida por los Estados receptores como una importante amenaza dada su naturaleza descontrolada y masiva (Kissinger; 2004:4).

RECONCEPTUALIZANDO LA SEGURIDAD: CAMBIO DE DILEMAS Y AMENAZAS 


\section{CONCLUSIONES}

En el contexto pos guerra fría, la concepción tradicional de la seguridad no es suficiente para explicar y a la vez comprender el auge de fenómenos que amenazan la estabilidad del sistema internacional, la integridad de los Estados y la seguridad de los individuos. Aunque el concepto de seguridad ligado a la defensa del Estado permite entender las acciones que algunos emprenden en contra de otros argumentando la defensa de sus valores e intereses nacionales, la reconfiguración del sistema internacional obliga a prestar atención a otras formas de conceptualización de término, más amplias en cuanto a actores, amenazas, y métodos de acción.

La emergencia en el escenario internacional de actores diferentes a los Estados e incluso el fortalecimiento de las capacidades de éstos en cuanto al alcance de sus acciones; el aumento de la interacción entre actores transnacionales, no estatales y estatales, y el auge de problemáticas de grupos humanos enteros referentes a la salud, la alimentación, el crimen, y la violencia interna, ponen de manifiesto la necesidad de repensar la seguridad en términos de su objeto o punto referente. Si bien el Estado continúa siendo protagonista de los procesos de reordenamiento del sistema mundial, son las comunidades y específicamente los hombres los que se han convertido en los destinatarios de una seguridad que tiene como prioridad mejorar sus condiciones de vida.

Es necesario por tanto seguir desarrollando nuevas perspectivas dentro del campo de la seguridad que se extiendan más allá de los límites que impone una visión militar tradicional, que tengan en cuenta la interdependencia de las relaciones entre los diferentes actores estatales y no estatales y que enfatice que el mundo no será un lugar seguro y «[pacífico] hasta que la gente tenga seguridad en su vida diaria» (Rojas; 2002).

Esta perspectiva ampliada de la seguridad, en la que ésta se concibe como un concepto y un valor multidimensional, implica que asuntos relacionados con el uso de la fuerza, la prevención de conflictos internos, el fortalecimiento de la cooperación internacional y las dimensiones cobijadas bajo la seguridad humana (la ecología, la economía, la sociedad, la política y la cultura) deben adquirir un papel central dentro de la agenda que rige las relaciones internacionales. El direccionamiento de la seguridad hacia problemas y asuntos que reflejan las condiciones de vulnerabilidad de la vida diaria de extensos grupos humanos alrededor del mundo, refuerza la idea que proyecta la necesidad de asegurar el nivel individual de seguridad para así posibilitar la seguridad estatal y la internacional.

Nos encontramos en una nueva etapa de la seguridad, que nos obliga a repensar las implicaciones del término no sólo frente a los aspectos militares, sino sobretodo frente a los aspectos de carácter político, social, económico y medioambiental, más aún cuando los Estados enfrentan serias dificultades para hacer frente a las amenazas de carácter no tradicional, anteriormente 
mencionadas y cuando presentan falencias estructurales de carácter institucional que les impide ser efectivos al momento de proveer condiciones mínimas básicas de desarrollo a sus poblaciones.

La agenda de seguridad, al tomar un giro hacia la dimensión humana, se compone de elementos ligados a las nociones de desarrollo de las que se desprenden a su vez las nociones de seguridad económica, seguridad alimentaria, seguridad comunitaria, seguridad personal, seguridad en salud, y seguridad política ciudadana.

Igualmente, los problemas que se encuentran detrás del concepto de seguridad humana siguen siendo asuntos de seguridad nacional, que trascienden a su vez el ámbito estatal para considerarse como asuntos de interés internacional, ya que la naturaleza de las amenazas desborda el ámbito geográfico delimitado por las fronteras nacionales. De ahí que no sólo el Estado debe proveer las condiciones de garantía de la seguridad individual, sino que la comunidad internacional tiene la «responsabilidad de proteger» el bienestar y la seguridad de poblaciones que se encuentran sometidas a gobiernos que violan sus derechos, a guerras genocidas y a crímenes de lesa humanidad, cuando sus propios Estados no tienen las capacidades o la voluntad para hacerlo.

Visiones no tradicionales a la seguridad, como el caso de la dimensión humana, «visualiza un nuevo orden global, un mundo único, fundado en un humanismo global. Lo central es resolver las necesidades básicas de la población en el contexto de la globalización y la interdependencia» (ROJAS; 2001: 23). Queda entonces planteado uno de los principales desafíos que enfrentan los Estados y más aún la comunidad internacional frente a una mayor cooperación y coordinación de políticas y la adopción de los mecanismos más efectivos para construir «un mundo libre de temor y necesidad».

\section{BIBLIOGRAFÍA}

- $\quad$ AGUIRRE, M (2000). «El debate sobre la seguridad en la globalización», en FASOC, Año 15, No 3, Julio- Septiembre.

- $\quad$ ALKIRE, S (2003). A conceptual Framework for Human Security. Working Paper No 2, Centre for Research on Inequality, Human Security and Ethnicity-CRISE, Queen Elizabeth House, University of Oxford.

- BIGO, D (2002). "Security and Inmigration: Toward a Critique of the Governmentality of Unease», en Global, Local, Political, Vol. 27.

- $\quad$ BAJPAI, K (2000). Human security: Concept and Measurement. Kroc Institute Occasional Paper \# 19:OP:1.

RECONCEPTUALIZANDO LA SEGURIDAD: CAMBIO DE DILEMASY AMENAZAS 
- $\quad$ BALDWIN, D (1997).»The concept of Security», en Review of International Studies, 23, 5- 26.

- DER DERIAN, J (2005). Global Security Manifesto, disponible en http:// www.watsonblogs,org/ globalsecurity/. Consultado el 5 de junio de 2008.

- $\quad$ FIDLER, D (2005).Transnational Threats to National Security: Daniel's Case against Linking Environmental Degradation and National Security. Princeton: Princeton Project on National Security.

- $\quad$ KISSINGER, A (2004). International Migration as a non-traditional security threat and the EU responses to this phenomenon. Warsaw: Central European Forum for Migration Research (CERFM), Working Paper.

- $\quad$ LIOTTA, P (2005). «Through the Looking Glass: Creeping Vulnerabilities and the Reordering of Security», en Security Dialogue, Vol 36, No 1, Marzo.

- MCRAE, R (2001). «Human Security in a Globalized World», en HUBERT, D. Human Security an the New Diplomacy. McGuill- Queen's Press.

- $\quad$ MEILAENDER, P (2001). Toward a Theory of Migration, cap 1. New York: Palgrave Macmillan, pp 9- 25 .

- $\quad$ MESAJASZ, C (2004). Security as an analytical concept. Paper presented at the 5th Pan- European conference on International relations, in The Hague, 9- 11 September.

- MORENO, J (2005). «La emergencia de una nueva lógica de seguridad política», en Colombia Internacional, Número 62 Julio- Diciembre.

- $\quad$ Programa de las Naciones Unidas para el Desarrollo (PNUD), Informe de Desarrollo Humano 1993.

- $\quad$ Programa de las Naciones Unidas para el Desarrollo (PNUD), 1994, Informe de Desarrollo Humano 1994, Nueva Cork, Oxford University Press.

- ROJAS ARAVENA, F (2000). «Repensando la seguridad en América Latina», en FASOC, Año 15, No 2, abril- junio.

(2001). «Seguridad humana: concepto emergente de la seguridad del S. XXl», en GOUCHA, M y ROJAS ARAVENA, F (Editores). Seguridad Humana, prevención de conflictos y paz en América Latina y el Caribe. Santiago. UNESCO/ FLACSO - Chile. 
- $\quad$ ROSENEAU, J (1992).»Governance, order and change in world politics», en ROSENEAU, J y CZEMPIEL, E (eds), Governance without government: Order and Change in World Politics, Cambridge University Press.

- $\quad$ SEN, A (2000). Why Human Security. Paper presented at the international symposium on human security, Tokyo, 28 July.

- $\quad$ TERRIFF, T, et. al. (2005). Security Studies Today. Londres: Polity.

- $\quad$ WILLIAMS, P (1999).»The Dark Side of Global Civil Society: The Role and Impact of transnational criminal organizations as a threat to International Security», en ALAGAPPA, M e INOGUCHI, T (eds), International Security Management and the United Nations. Japón: United Nations University Press.

- $\quad$ ZINDER, C (1999). Contemporary Security and statecraft. Londres: Routledge. 